\title{
Effect of treatment with botulinum toxin on neurogenic blepharospasm
}

\author{
J S ELSTON, R W ROSS RUSSELL
}

\begin{abstract}
Botulinum toxin type A creates temporary localised flaccid paralysis after injection into skelet:al muscle. Thirty four patients with blepharospasm, of whom 28 also had the oromandibular dystonia syndrome, were treated with injections of botulinum toxin type $A$ into the orbicularis oculi, and 28 showed functional improvement after the treatment. A high incidence of local side effects occurred, especially partial ptosis, which was well tolerated. There were no systemic side effects. The average period of relief was 2.5 months, increasing to 2.8 months after a second injection. Functional improvement was limited in patients with severe associated dystonia.
\end{abstract}

\section{Introduction}

Idiopathic blepharospasm consists of repeated involuntary spasms of both orbicularis oculi muscles, sometimes spreading to other parts of the face. ${ }^{1}$ Of insidious onset it usually occurs in the fifth to seventh decade and if untreated progresses to functional blindness, associated with disabling and disfiguring spasms of the muscles of the face and jaw (oromandibular dystonia). ${ }^{23}$ Although formerly regarded as a psychogenic disorder, the syndrome is most probably due to organic dysfunction of the basal ganglia ${ }^{3-5}$ or, rarely, the rostral brain stem. ${ }^{6}$ Blepharospasm may also occur in extrapyramidal disorders such as parkinsonism, Huntington's disease, progressive supranuclear palsy, and tardive dyskinesia induced by drugs. No consistent or sustained response occurs with drugs such as levodopa or benzhexol, ${ }^{7}$ although tetrabenazine may have some effect. ${ }^{8}$ Mechanical treatments such as ptosis props or strips of adhesive are seldom effective. Blepharospasm may be treated surgically by bilateral partial avulsion of the facial nerve, ${ }^{8}$

Moorfields Eye Hospital, London EC1V 2PD

J S ELSTON, FRCS, lecturer in clinical ophthalmology

R W ROSS RUSSELL, DM, FRCP, consultant neurologist

Correspondence to: $\mathrm{Mr}$ Elston. but there is a $25 \%$ chance of recurrence and further surgery on the eyelid for ectropion is frequently required ${ }^{10}$; inadvertent denervation of the buccolabial musculature interferes with eating, and the facial appearance is altered. Stripping of the orbicularis muscle as described by Gillum and Anderson causes fewer complications and less cosmetic disturbance. ${ }^{11}$

Botulinum toxin type A blocks the release of acetylcholine from motor nerve terminals. ${ }^{12}{ }^{13}$ It can be accurately measured, and small doses injected directly into muscle produce a localised flaccid paralysis without systemic toxicity. ${ }^{14}$ Function of the muscle returns when new end plates are formed by sprouting from axons, ${ }^{15} 16$ but this process takes many weeks. Repeated injections are possible as the doses used are below the threshold of recognition by the immune system. ${ }^{17}$ Injection of botulinum toxin $\mathrm{A}$ into the orbital and palpebral orbicularis oculi can therefore be used to relieve blepharospasm. ${ }^{17}{ }^{18} \mathrm{We}$ report the results of treatment with botulinum toxin $A$ in 34 patients with blepharospasm.

\section{Patients and methods}

Idiopathic blepharospasm was diagnosed clinically in 34 patients (12 men, 22 women; mean age 64 (range 38-78). The mean duration of their history was 4.1 years (range 8 months to 10 years). Six patients had blepharospasm alone, but 28 also had oromandibular dystonia. Of these, 11 had involuntary spasms extending to the neck muscles (10 having antecollis and one retrocollis), three had pharangeal spasms, and one had spasmodic dysphonia. Three patients had benign essential tremor of the arms, two had Parkinson's disease, one had progressive supranuclear ophthalmoplegia, and one had writer's cramp. In three cases a previous episode of dystonia had resolved spontaneously. Four patients had a family history of focal dystonia (first degree relatives). None of the patients had responded to a wide range of drugs, and five had undergone surgery (avulsion of the facial nerve in four (one twice) and stereotactic neurosurgery in one).

The degree of disability was assessed on a functional scale, particular attention being paid to the patient's ability to work, drive, go about outdoors alone, read, and watch television. The percentage of time during a typical day when the eyes were closed by blepharospasm, was calculated and the patients categorised as functionally blind (eyes shut for over $80 \%$ of the day; $n=23$ ), disabled (eyes shut for $30-80 \%$ of the day; $\mathrm{n}=10$ ), or inconvenienced (eyes shut for $10-30 \%$ of the day; $n=1$ ). We used an identical scale for assessing the effects of treatment. 
Botulinum toxin $\mathrm{A}$ in ampoules containing $0.05 \mu \mathrm{g}$ freezedried neurotoxin bound to haemagglutinin was reconstituted with $1.0 \mathrm{ml}$ sterile saline devoid of preservative and then diluted to give $5 \mathrm{ng}$ in $2 \mathrm{ml}$ saline. The orbital and preseptal orbicularis oculi were injected with $0.4 \mathrm{ml}$ laterally and medially in the lower lid and $0.6 \mathrm{ml}$ in two doses avoiding the centre of the upper lid. We initially used botulinum toxin A supplied by Professor Schantz of the University of Wisconsin; we subsequently used botulinum toxin A supplied by Professor Melling of the Vaccine Research and Production Laboratory, Porton Down, at the same dose. Although the median lethal doses of these two preparations had been found to be the same in mice, we later found that the botulinum toxin A from Porton Down was more neurotoxic in man and so we reduced the dose, a total of $1.50 \mathrm{ng}$ in $2 \mathrm{ml}$ saline being injected around each eye. The patients were re-examined one week after the initial injection, when further treatment was given if necessary and any side effects noted. The patients were then seen at two, four, and eight weeks aftter treatment or at any stage if the blepharospasm returned. functionally blind. The relief of blepharospasm enabled 18 to resume a normal life; this group included all those who had blepharospasm alone and all those who had had previous operations. Nine had oromandibular dystonia, and most reported some improvement in these symptoms when the blepharospasm was relieved. A second group of 10 patients showed functional improvement after treatment with botulinum toxin $\mathrm{A}$ although six remained disabled by neck, jaw, or pharyngeal spasms.

There was little difference, therefore, in the response to treatment between those with blepharospasm alone and those with more complex dystonias. In those with more complex dystonias local relief of spasms was obtained in the orbicularis oculi but other spasms continued as before. These cases represent the most severe end of the range of focal dystonia of adult onset, ${ }^{14}$ and it is not possible to help them with any other form of treatment. ${ }^{2}$ Of the other patients studied, three with symp-

Summary of treatment after initial injections

\begin{tabular}{|c|c|c|c|c|c|c|c|}
\hline \multirow[b]{2}{*}{ Clinical state } & \multirow[b]{2}{*}{$\mathbf{n}$} & \multicolumn{4}{|c|}{ Functional category after initial injection ${ }^{*}$} & \multirow[b]{2}{*}{ Subsequent treatment } & \multirow[b]{2}{*}{$\mathbf{n}$} \\
\hline & & Blind & Disabled & Inconvenienced & Normal & & \\
\hline $\begin{array}{l}\text { Relief of symptoms } \\
\text { Improvement } \\
\text { No change } \\
\text { Worse }\end{array}$ & $\begin{array}{r}18 \\
10 \\
1 \\
5\end{array}$ & $\begin{array}{l}1 \\
4\end{array}$ & $\begin{array}{l}4 \\
1\end{array}$ & 6 & 18 & $\begin{array}{l}\text { Repeat injections } \\
\text { Repeat injections } \\
\text { Facial nerve surgery } \\
\text { Repeat injections }\end{array}$ & $\begin{array}{r}18 \\
7 \\
1 \\
3\end{array}$ \\
\hline
\end{tabular}

*See Patients and methods for definitions.

\section{Results}

After a latent period of $\mathbf{4 8}$ hours the intensity of the blepharospasm lessened progressively over five or six days. Eighteen patients had complete relief, the condition of 10 improved, in one no change occurred, and five were functionally worse due to side effects. A second set of injections one or two weeks after the first, usually to the brows nasally and temporally, was required in 10 of the 28 patients who had benefited from the initial treatment. Side effects were common, occurring in 15 of the patients: most were mild, comprising partial ptosis or minimal imbalance of the ocular muscle resulting in diplopia in certain positions of gaze, but the patients considered their condition to have improved compared with that before treatment. Five patients were more disabled: in one there was rapid resolution of a weakness of the superior rectus muscles, but complete bilateral ptosis with generalised weakness of the extraocular muscles developed in the first four patients treated after the supplier of botulinum toxin A had been changed. Two were over 75 years of age and took an average of 11 weeks to recover normal opening of the lids and ocular motility, while two patients aged 57 and 68 took five weeks to recover. Subsequently relief of blepharospasm averaged six weeks in these four patients.

Symptomatic improvement or relief lasted on average 2.55 months after the first injections but appreciably longer ( $5 \cdot 25$ months) in the four patients who had had recurrent spasms after avulsion of the facial nerve. Four patients who thought that the initial injections had been of marginal or no benefit subsequently underwent bilateral avulsion of the facial nerve, and 28 received further injections (table). In 27 patients the functional improvement was maintained. Over six months an average of 2.5 sets of injections were required, and relief after the second injections lasted on average 2.8 months. One patient suffered recurrent partial ptosis and diplopia and declined further injections; six remained functionally disabled by oromandibular dystonia or neck spasms despite relief of blepharospasm. Twenty two of the 34 patients were either able to function normally (18) or improved (four) with treatment: many were able to resume work, driving, reading, and a normal social life.

\section{Discussion}

The clinical features of these patients emphasise that there is a continuum from blepharospasm alone, through blepharospasm plus the oromandibular dystonia syndrome, to those who, in addition, have pharyngeal spasms, spasmodic dysphonia, retrocollis, or writer's cramp. All patients were adults, usually in their seventh decade, with more women than men. Most were toms that recurred soon after the injections subsequently underwent bilateral avulsion of the facial nerve. The fourth patient who was treated surgically suffered a severe anxiety state and received no benefit from one set of injections.

The most common side effect was a $2-3 \mathrm{~mm}$ asymmetric bilateral ptosis caused by spread of the toxin to the levator palpebrae superioris, which appears to be particularly sensitive to the toxin. As it begins in the anterior medial part of the orbit the inferior oblique muscle is also susceptible to the spread of toxin from the orbicularis, producing vertical strabismus. In 11 patients the side effects resolved over two to four weeks, and even after a fourfold overdose of neurotoxin in four patients recovery, although taking longer, especially in the older patients, was complete, with relief of blepharospasm.

Relief of blepharospasm from the first injections in the 28 patients who received second injections was on average 2.55 months: in the four patients who had undergone avulsion of the facial nerve it was 5.25 months, suggesting that the sprouting of new motor end plates in the regenerated motor nerve axon was defective. Experiments on animals indicate that a permanent change in the pattern of motor innervation of skeletal muscle occurs after recovery from an injection of botulinum toxin A. Possibly, repeated injections may lead to progressive disruption of the orbicularis innervation and an increased period of freedom from symptoms. ${ }^{19}$

The incidence of side effects from the second injections was lower than from the first ( 10 patients with mild ptosis including two with transient diplopia), with recovery after two to three weeks. One patient, however, withdrew from the trial after recurrent diplopia, but 27 had a third injection after an average of 2.8 months.

We conclude that treatment of blepharospasm and the oromandibular dystonia syndrome with botulinum toxin A is simple and effective and free of systemic or severe local side effects. It is particularly useful in patients with blepharospasm alone and those with recurrent spasm after avulsion of the facial nerve, when normal visual function is restored. In the presence of severe oromandibular, neck, or pharyngeal spasms the relief of blepharospasm is insufficient to restore normal function. An average of five sets of injections annually would probably be necessary to avoid recurrence of symptoms. The treatment has also been applied to four patients with hemifacial spasm and one with facial synkinesis after facial nerve palsy. Relief of symptoms 
occurred in all cases. Injections of botulinum toxin A might, therefore, be extended to the treatment of other groups of muscles affected by spasmodic dystonia.

We thank Professor W I McDonald, Professor C D Marsden, and Professor A C Bird for referring patients and Miss Karen Betts for secretarial help.

\section{References}

1 Jan Kovic J, Havins WE, Wilkins RB. Blinking and blepharospasm: mechanisms, diagnosis, management. $\mathcal{J} A M A$ 1982;248:3160-4.

Ophthalmol Soc 1956;54:

453-519.
Marsden CD. Blepharospasm-oromandibular dystonia syndrome (Brueghel's syndrome). F Neurol Neurosurg Psychiatry 1976;39:1204-9.

Jan Kovic J, Ford J. Blepharospasm of orofacial-cervical dystonia: clinical and Casey DE Pharmacology of blepharospasm-oromandibular dystonia syndrome. Neurology 1980;30:690-5.

Jan Kovic J, Patel SC. Blepharospasm associated with brain stem lesions. Neurology $1983 ; 33: 1237-40$.

7 Skarf B, Sharp JA. Choline for blepharospasm. N Engl f Med 1981;305:957-8.
8 Bird AC, MacDonald WI. Essential blepharospasm. Trans Ophthalmol Soc UK 975;95:250-3.

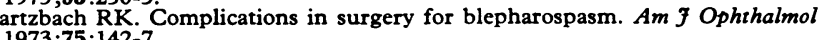
Cord CD, Coles

CW Shore JW, Spector R, Putman JR. Treatment of essential blepharospasm. Arch Ophthalmol 1984;102:266-73.

11 Gillum WN, Anderson RL. Blepharospasm surgery: an anatomical approach. Arch Ophthalmol 1981;99:1056-62.

12 Duchen LW, Strich SJ. Changes in the pattern of motor innervation of skeletal muscle in the mouse after local injection of Clostridium botulinum toxin. Physiol (Lond) 1967;189:2-4

13 Boroff DA, del Castillo J, Evoy WH, Steinherdt RA. Observations on the action of type A botulinum toxin on frog neuromuscular junctions. I Physiol (Lond)

14 Guyton AC, MacDonald MA. Physiology of botulinum toxin. Archives of Neurology and Psychiatry 1947;57:578-92. toxin in the motor end plates of slow snd fast skeletal muscle fibres of the mouse. $\exists$ Neurol Sci 1971;14:47-60.

16 Duchen LW. Changes in the electron microscopic structure of slow and fast skeletal muscle fibres of the mouse after the local injection of botulinum toxin. f Neurol Sci 1971;14:60-74.

17 Scott AB. Botulinum toxin injection into extraocular muscles as an alternative to strabismus surgery Ophthalmology 1980;87:1044-7. ruch BR, Felt DP, Woino TH, Musch DC. Treatment of blepharospasm with
botulinum toxin. Arch Ophthalmol 1984;102:1464-8.

19 Tonge DA. Chromic effects of botulinum toxin on neuromuscular transmission and sensitivity to acetylcholine in slow and fast skeletal muscle of the mouse. f Physiol (Lond) 1974;241:127-39.

(Accepted 25 March 1985)

\title{
Relation between consumption of alcohol and fatty acids esterifying serum cholesterol in healthy men
}

\author{
JEAN-MICHEL WARNET, FRANÇOIS CAMBIEN, VIVIANE VERNIER, MARTINE PECORARO, \\ CLAUDIE FLAMENT, PIERRE DUCIMETIERE, ALAIN JACQUESON, \\ JACQUES-LUCIEN RICHARD， JEAN-ROGER CLAUDE
}

\begin{abstract}
The relation between consumption of alcohol (established by interview), two of its typical markers ( $\gamma$-glutamyltransferase activity and mean corpuscular volume), and the composition of fatty acids in plasma cholesterol esters was investigated in an epidemiological study of 1467 actively employed men aged 35-45. In this group of subjects mean consumption of alcohol was 34.6 (SD 25.2) g/day. Palmitoleic acid and oleic acid were positively correlated with consumption of alcohol, $\gamma$ glutamyltransferase activity, and mean corpuscular volume, while linoleic acid was negatively correlated with these variables. When these three fatty acids, $\gamma$-glutamyltransferase activity, mean corpuscular volume, and the fat content of the diet were introduced into a multivariate regression analysis, with consumption of alcohol as the dependent variable, only palmitoleic
\end{abstract}

Groupe d'Etude sur l'Epidémiologie de l'Athérosclérose, 75017 Paris, France

JEAN-MICHEL WARNET, PHD, professor

FRANCOIS CAMBIEN, MD

MARTINE PECORARO, PHD

PIERRE DUCIMETIERE, PHD

ALAIN JACQUESON, PHD

JACQUES-LUCIEN RICHARD, MD

JEAN-ROGER CLAUDE, PHD

Laboratoire de la Société Astra-Calvé, 92601 Asnières

VIVIANE VERNIER, technician

Unité 1 de l'Institut National de la Santé et de la Recherche Médicale, 75877 Paris Cedex 18

CLAUDIE FLAMENT, technician

Correspondence to: Professor Jean-Michel Warnet, Laboratoire de Recherches sur les Maladies Cardio-Vasculaires de la Direction des Affaires Sanitaires et Sociales de Paris, 88 Rue de la Jonquière, 75017 Paris, France. acid, $\gamma$-glutamyltransferase activity, mean corpuscular volume, and the monounsaturated fat content of the diet remained significant.

Palmitoleic acid seems to be an independent correlate of consumption of alcohol and could be useful in epidemiological and clinical studies as a variable of consumption.

\section{Introduction}

Studies in both animals ${ }^{1-3}$ and $\operatorname{man}^{4-7}$ have shown that consumption of alcohol, either acute or chronic, strongly modifies the pattern of fatty acids in the liver and serum lipids. In particular, a decrease in the proportion of hepatic and serum linoleic acid has been reported repeatedly in alcoholics. ${ }^{4-7}$ No epidemiological data have been available concerning this relation despite its possible importance in the association between coronary heart disease and the intake of alcohol and linoleic acid, which has been reported in some epidemiological and clinical studies. ${ }^{8-11}$

This paper reports the association observed between consumption of alcohol, two of its biological markers ( $\gamma$-glutamyltransferase activity and mean corpuscular volume), and the composition of fatty acids in plasma cholesterol esters in a group of apparently healthy men at baseline of the Paris prospective study II.

\section{Subjects and methods}

The reported results concern the cross sectional data obtained at entry into the Paris prospective study II from a subgroup of 1467 men aged 35-45. This study on cardiovascular risk factors began in 1982 , but follow up data were not yet available. The subjects of this study are male employees of a large public organisation in Paris, who undergo regular medical examinations. Each subject answered 